\title{
Decomposition of Generalized Mittag-Leffler Function and Its Properties
}

\author{
Jyotindra C. Prajapati ${ }^{1}$, Ajay Kumar Shukla ${ }^{2}$ \\ ${ }^{1}$ Department of Mathematical Sciences, Faculty of Applied Sciences, Charotar University of Science and Technology, Anand, India \\ ${ }^{2}$ Department of Mathematics, S.V. National Institute of Technology, Surat, India \\ Email: \{jyotindra18, ajayshukla2\}@rediffmail.com
}

Received August 16, 2011; revised September 26, 2011; accepted October 10, 2011

\section{ABSTRACT}

The principal aim of the paper is devoted to the study of some special properties of the function $E_{\alpha, \beta}^{\gamma, q}(z)$ for $\alpha=\frac{1}{n}$. Authors defined the decomposition of the function $E_{\alpha, \beta}^{\gamma, q}(z)$ in the form of truncated power series as Equations (1.7), (1.8) and their various properties including integral representation, derivative, inequalities and their several special cases are obtained. Some new results are also established for the function $E_{\alpha, \beta}^{\gamma, q}(z)$.

Keywords: Generalized Mittag-Leffler Function; Beta Function; Gamma Function; Integral Representation

\section{Introduction}

In 1903, the Swedish mathematician Gosta Mittag-Leffler introduced the function $E_{\alpha}(z)$ (Gorenflo et al. [1]) defined as,

$$
E_{\alpha}(z)=\sum_{n=0}^{\infty} \frac{z^{n}}{\Gamma(\alpha n+1)},
$$

where $z$ is a complex variable and $\Gamma(\cdot)$ is a gamma function, $\alpha \geq 0$. The Mittag-Leffler function is the direct generalisation of the exponential function to which it reduces for $\alpha=1$. For $0<\alpha<1$, it interpolates between the pure exponential and a hypergeometric function $\frac{1}{1-z}$. Mittag-Leffler function naturally occurs as the solution of the fractional order differential equations and (or) fractional order integral equations.

Wiman [2] studied the generalisation of $E_{\alpha}(z)$, that is given by

$$
\begin{gathered}
E_{\alpha, \beta}(z)=\sum_{n=0}^{\infty} \frac{z^{n}}{\Gamma(\alpha n+\beta)}, \\
(\alpha, \beta \in \mathbb{C} ; \operatorname{Re}(\alpha)>0, \operatorname{Re}(\beta)>0) .
\end{gathered}
$$

which is known as Wiman's function.

Prabhakar [3] investigated the function $E_{\alpha, \beta}^{\gamma}(z)$ as

$$
\begin{gathered}
E_{\alpha, \beta}^{\gamma}(z)=\sum_{n=0}^{\infty} \frac{(\gamma)_{n}}{\Gamma(\alpha n+\beta)} \frac{z^{n}}{n !}, \\
(\alpha, \beta, \gamma \in \mathbb{C} ; \operatorname{Re}(\alpha)>0, \operatorname{Re}(\beta)>0, \operatorname{Re}(\gamma)>0) .
\end{gathered}
$$

Gorenflo et al. ([4]), Kilbas and Saigo [5], Kilbas et al. [6], Miller [7], Saigo and Kilbas [8] have studied several properties and applications of (1.1)-(1.3).

Recently, Shukla and Prajapati [9] introduced the function $E_{\alpha, \beta}^{\gamma, q}(z)$, which is defined for $\alpha, \beta, \gamma \in \mathbb{C}$; $\operatorname{Re}(\alpha)>0, \operatorname{Re}(\beta)>0, \operatorname{Re}(\gamma)>0$ and $q \in(0,1) \cup \mathbb{N}$ by:

$$
E_{\alpha, \beta}^{\gamma, q}(z)=\sum_{n=0}^{\infty} \frac{(\gamma)_{q n}}{\Gamma(\alpha n+\beta)} \frac{z^{n}}{n !},
$$

where $(\gamma)_{q n}=\frac{\Gamma(\gamma+q n)}{\Gamma(\gamma)}$ denotes the generalized Pochhammer symbol which, in particular, reduces to $q^{q n} \prod_{r=1}^{q}\left(\frac{\gamma+r-1}{q}\right)_{n}$ if $q \in \mathbb{N}$.

For $\beta=\gamma=q=1,(1.4)$ reduces to (1.1) which was studied by Mittag-Leffler in terms of its applications to the theory of entire functions.

Incidentally, (1.4) is generalization of the exponential function $e^{z}$, the confluent hypergeometric function $\Phi(\gamma, \alpha ; z)$ and the functions which are defined in (1.1)(1.3).

Ikehata and Siltanen [10] defined truncated power series of $E_{\alpha}(z)$ as,

$$
E_{\frac{1}{n}}^{n N}(z)=\sum_{m=0}^{N} \frac{z^{n m}}{m !}+\sum_{m=0}^{N-1} \sum_{j=1}^{n-1} \frac{z^{n m+j}}{\Gamma\left(\frac{n m+j}{n}+1\right)} ;
$$




$$
E_{\frac{1}{n}}(z)=\sum_{m=0}^{\infty} \frac{z^{n m}}{m !}+\sum_{m=0}^{\infty} \sum_{j=1}^{n-1} \frac{z^{n m+j}}{\Gamma\left(\frac{n m+j}{n}+1\right)} .
$$

Whereas in [10], they have used the functions (1.5) and (1.6) in the study of electrical impedance tomography.

In this paper, we have defined the decomposition of the function $E_{\alpha, \beta}^{\gamma, q}(z)$ in the form of truncated power series

$$
\begin{aligned}
E_{\frac{1}{n}, \beta}^{\gamma, q, n N}(z)= & \sum_{m=0}^{N} \frac{(\gamma)_{q m} z^{n m}}{\Gamma(m+\beta) m !} \\
& +\sum_{m=0}^{N-1} \sum_{j=1}^{n-1} \frac{(\gamma)_{q m} z^{n m+j}}{\Gamma(m+\beta) \Gamma\left(\frac{n m+j}{n}+1\right)}
\end{aligned}
$$

and as a special case for $\alpha=\frac{1}{n}$ :

$$
\begin{aligned}
E_{\frac{1}{n}, \beta}^{\gamma, q}(z)= & \sum_{m=0}^{\infty} \frac{(\gamma)_{q m} z^{n m}}{\Gamma(m+\beta) m !} \\
& +\sum_{m=0}^{\infty} \sum_{j=1}^{n-1} \frac{(\gamma)_{q m} z^{n m+j}}{\Gamma(m+\beta) \Gamma\left(\frac{n m+j}{n}+1\right)}
\end{aligned}
$$

where $n \geq 2, \quad N \geq 1 ; \beta, \gamma \in \mathbb{C} ; \operatorname{Re}(\beta)>0, \operatorname{Re}(\gamma)>0$ and $q \in(0,1) \cup \mathbb{N}$.

(1.5) and (1.6) are special cases of (1.7) and (1.8), given by

$$
E_{\frac{1}{n}, 1}^{1,1, n N}(z)=E_{\frac{1}{n}}^{n N}(z) \text { and } E_{\frac{1}{n}, 1}^{1,1}(z)=E_{\frac{1}{n}}(z) .
$$

In what follows are known formulae, those used studying properties of the functions

$$
E_{\alpha, \beta}^{\gamma, q}(z), E_{\frac{1}{n}, \beta}^{\gamma, q, n N}(z) \text { and } E_{\frac{1}{n}, \beta}^{\gamma, q}(z) .
$$

The Beta function (Rainville [11]) is defined by,

$$
B(a, b)=\int_{0}^{1} z^{a-1}(1-z)^{b-1} \mathrm{~d} z,
$$$$
\text { where } \operatorname{Re}(a)>0, \operatorname{Re}(b)>0 .
$$

The Gamma function (Rainville [11]) is defined by,

$$
\Gamma(z)=\int_{0}^{\infty} e^{-t} t^{z-1} \mathrm{~d} t, \text { where } \operatorname{Re}(z)>0 .
$$

The relation between Beta and Gamma functions is

$$
B(a, b)=\frac{\Gamma(a) \Gamma(b)}{\Gamma(a+b)},
$$

$$
\text { where } \operatorname{Re}(a)>0, \operatorname{Re}(b)>0 .
$$

The Error function (Rainville [11]) is defined by,

$$
\operatorname{erf}(z)=\frac{2}{\sqrt{\pi}} \int_{0}^{z} \exp \left(-t^{2}\right) \mathrm{d} t
$$

The complementary Error function (Rainville [11]) is defined by,

$$
\operatorname{erf}_{c}(z)=1-\operatorname{erfc}(z)=\frac{2}{\sqrt{\pi}} \int_{z}^{\infty} \exp \left(-t^{2}\right) \mathrm{d} t .
$$

Legendre's duplication formula (Rainville [11]) is

$$
\sqrt{\pi} \Gamma(2 z)=2^{2 z-1} \Gamma(z) \Gamma\left(z+\frac{1}{2}\right) .
$$

The Laplace transform (Sneddon [12]) of the function $f(z)$ is defined as,

$$
L\{f(z)\}=\int_{0}^{\infty} e^{-s z} f(z) \mathrm{d} z, \text { where } \operatorname{Re}(s)>0 .
$$

Ikehata and Siltanen [10] used following inequality,

$$
\left|\sum_{m=N+1}^{\infty} \frac{z^{m}}{m !}\right| \leq \frac{|z|^{N+1} e^{|\operatorname{Re} z|}}{(N+1) !}, \text { where } N \geq 1 .
$$

\section{Main Results}

THEOREM 1. Integral representations of the function $E_{\frac{1}{n}, \beta}^{\gamma, q}(z)$ is given by

$$
\begin{aligned}
E_{\frac{1}{n}, \beta}^{\gamma, q}(z)= & E_{1, \beta}^{\gamma, q}\left(z^{n}\right) \\
& +n \sum_{k=1}^{n-1} \frac{1}{\Gamma\left(1-\frac{k}{n}\right)} \int_{0}^{z} E_{1, \beta}^{\gamma, q}\left(z^{n}-u^{n}\right) u^{n-k-1} \mathrm{~d} u,
\end{aligned}
$$

where $n \geq 2, \beta, \gamma \in \mathbb{C} ; \operatorname{Re}(\beta)>0, \operatorname{Re}(\gamma)>0$ and $q \in(0,1) \cup \mathbb{N}$.

Proof. Consider the integral,

$$
\int_{0}^{z} E_{1, \beta}^{\gamma, q}\left(z^{n}-u^{n}\right) u^{n-k-1} \mathrm{~d} u
$$

Substituting $u=z w$ and using (1.4), we get

$$
=\int_{0}^{1} \sum_{m=0}^{\infty} \frac{(\gamma)_{q m} z^{n m}\left(1-w^{n}\right)^{m}}{\Gamma(m+\beta) m !}(z w)^{n-k} \mathrm{~d} w .
$$

Using (1.10), the above equation reduces to

$$
=\sum_{m=0}^{\infty} \frac{(\gamma)_{q m} z^{n m+n-k}}{\Gamma(m+\beta) m !} \frac{B\left(m+1,1-\frac{k}{n}\right)}{n}
$$

and use of (1.12), yields

$$
\begin{aligned}
& \int_{0}^{z} E_{1, \beta}^{\gamma, q}\left(z^{n}-u^{n}\right) u^{n-k-1} \mathrm{~d} u \\
& =\sum_{m=0}^{\infty} \frac{(\gamma)_{q m} z^{n m+n-k}}{\Gamma(m+\beta)} \frac{\Gamma\left(1-\frac{k}{n}\right)}{n \Gamma\left(\frac{n m+n-k}{n}+1\right)} .
\end{aligned}
$$


i.e.

$$
\sum_{m=0}^{\infty} \frac{(\gamma)_{q m} z^{n m+n-k}}{\Gamma(m+\beta) \Gamma\left(\frac{n m+n-k}{n}+1\right)}=\frac{n}{\Gamma\left(1-\frac{k}{n}\right)} \int_{0}^{z} E_{1, \beta}^{\gamma, q}\left(z^{n}-u^{n}\right) u^{n-k-1} \mathrm{~d} u .
$$

Taking summation over $k=1$ to $k=n-1$ and using (1.8), we arrive at

$$
E_{\frac{1}{n}, \beta}^{\gamma, q}(z)-E_{1, \beta}^{\gamma, q}\left(z^{n}\right)=n \sum_{k=1}^{n-1} \frac{1}{\Gamma\left(1-\frac{k}{n}\right)} \int_{0}^{z} E_{1, \beta}^{\gamma, q}\left(z^{n}-u^{n}\right) u^{n-k-1} \mathrm{~d} u .
$$

This completes the proof of the theorem.

THEOREM 2. If $n \geq 2, \beta, \gamma \in \mathbb{C} ; \operatorname{Re}(\beta)>0, \operatorname{Re}(\gamma)>0$ and $q \in(0,1) \cup \mathbb{N}$, then

$$
\frac{\mathrm{d}}{\mathrm{d} z}\left[z^{\beta-1} E_{\frac{1}{n}, \beta}^{\gamma, q}\left(z^{\frac{1}{n}}\right)\right]=z^{\beta-1}\left[\sum_{k=1}^{n-1} \frac{(\gamma)_{q(n-k)} z^{-\frac{k}{n}}}{\Gamma\left(-\frac{k}{n}+\beta\right)(n-k) !}+\sum_{k=1}^{n-1} \frac{(\gamma)_{q(m+n)} z^{\frac{m}{n}}}{\Gamma\left(\frac{m}{n}+\beta\right)(m+n) !}\right]
$$

Proof. Applying (1.4) in left-hand side of (2.2), we obtain

$$
\frac{\mathrm{d}}{\mathrm{d} z}\left[z^{\beta-1} E_{\frac{1}{n}, \beta}^{\gamma, q}\left(z^{\frac{1}{n}}\right)\right]=\sum_{m=1}^{\infty} \frac{(\gamma)_{q m} z^{\frac{m}{n}+\beta-2}}{\Gamma\left(\frac{m}{n}+\beta-1\right) m !}=\sum_{m=1}^{n-1} \frac{(\gamma)_{q m} z^{\frac{m}{n}+\beta-2}}{\Gamma\left(\frac{m}{n}+\beta-1\right) m !}+\sum_{m=n}^{\infty} \frac{(\gamma)_{q m} z^{\frac{m}{n}+\beta-2}}{\Gamma\left(\frac{m}{n}+\beta-1\right) m !} .
$$

Setting $m=n-k$ in the first summation and replacing $m$ by $m+n$ in second, right-hand side of (2.3) reduces to

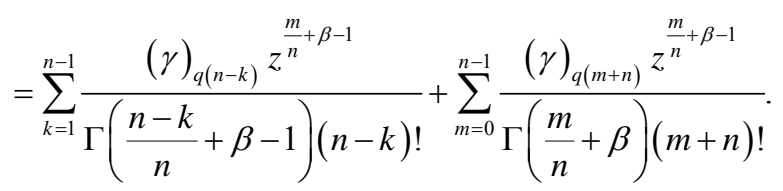

This completes the proof of the theorem.

Remark on Theorem 2. If $\gamma=q=1$, then Theorem 2 leads a particular case of (2.3)

$$
\frac{\mathrm{d}}{\mathrm{d} z}\left[z^{\beta-1} E_{\frac{1}{n}, \beta}\left(z^{\frac{1}{n}}\right)\right]=z^{\beta-1}\left[\sum_{k=1}^{n-1} \frac{z^{-\frac{k}{n}}}{\Gamma\left(-\frac{k}{n}+\beta\right)}+E_{\frac{1}{n}, \beta}\left(z^{\frac{1}{n}}\right)\right],
$$

where $n \geq 2, \beta, \gamma \in \mathbb{C} ; \operatorname{Re}(\beta)>0, \operatorname{Re}(\gamma)>0$ and $q \in(0,1) \cup \mathbb{N}$.

THEOREM 3. If $n \geq 2, \beta, \gamma \in \mathbb{C} ; \operatorname{Re}(\beta)>0, \operatorname{Re}(\gamma)>0$ and $q \in(0,1) \cup \mathbb{N}$.

$$
\left|E_{\frac{1}{n}, \beta}^{\gamma, q}(z)\right| \leq \sum_{k=0}^{n-1} \frac{|z|^{k}}{\Gamma\left(1+\frac{k}{n}\right)} E_{1, \beta}^{\gamma, q}\left(\left|\operatorname{Re} z^{n}\right|\right) .
$$

Proof. Substituting $u=z w$ in (2.1), we get

$$
E_{\frac{1}{n}, \beta}^{\gamma, q}(z)=E_{1, \beta}^{\gamma, q}\left(z^{n}\right)+n \sum_{k=1}^{n-1} \frac{z^{n-k}}{\Gamma\left(1-\frac{k}{n}\right)} \int_{0}^{1} E_{1, \beta}^{\gamma, q}\left(z^{n}\left(1-w^{n}\right)\right) w^{n-k-1} \mathrm{~d} w .
$$

Therefore,

$$
\left|E_{\frac{1}{n}, \beta}^{\gamma, q}(z)\right| \leq E_{1, \beta}^{\gamma, q}\left(\left|\operatorname{Re} z^{n}\right|\right)+n \sum_{k=1}^{n-1} \frac{|z|^{n-k}}{\Gamma\left(1-\frac{k}{n}\right)} E_{1, \beta}^{\gamma, q}\left(\left|\operatorname{Re} z^{n}\right|\right) \int_{0}^{1} w^{n-k-1} \mathrm{~d} w .
$$


The simplification of the above inequality gives,

$$
\left|E_{\frac{1}{n}, \beta}^{\gamma, q}(z)\right| \leq\left[1+\sum_{k=1}^{n-1} \frac{|z|^{n-k}}{\Gamma\left(1-\frac{k}{n}\right)} \frac{1}{\left(1-\frac{k}{n}\right)}\right] E_{1, \beta}^{\gamma, q}\left(\left|\operatorname{Re} z^{n}\right|\right)=\left[1+\sum_{k=1}^{n-1} \frac{|z|^{n-k}}{\Gamma\left(\frac{n-k}{n}+1\right)}\right] E_{1, \beta}^{\gamma, q}\left(\left|\operatorname{Re} z^{n}\right|\right)
$$

i.e.

$$
\left|E_{\frac{1}{n}, \beta}^{\gamma, q}(z)\right| \leq\left[1+\sum_{k=1}^{n-1} \frac{|z|^{k}}{\Gamma\left(\frac{k}{n}+1\right)}\right] E_{1, \beta}^{\gamma, q}\left(\left|\operatorname{Re} z^{n}\right|\right) .
$$

This proves the theorem.

Remark on Theorem 3: It is easy to verify the following inequality,

$$
\left|E_{\frac{1}{n}, \beta}^{\gamma, q, n N}(z)\right| \leq E_{\frac{1}{n}, \beta}^{\gamma, q, n N}(|z|) \leq E_{\frac{1}{n}, \beta}^{\gamma, q}(|z|) \leq \sum_{k=0}^{n-1} \frac{|z|^{k}}{\Gamma\left(1+\frac{k}{n}\right)} E_{1, \beta}^{\gamma, q}\left(|z|^{n}\right) .
$$

THEOREM 4. If $n \geq 2, \beta, \gamma \in \mathbb{C} ; \operatorname{Re}(\beta)>0, \operatorname{Re}(\gamma)>0$ and $q \in(0,1) \cup \mathbb{N}$, then

$$
\left|E_{\frac{1}{n}, \beta}^{\gamma, q}(z)-E_{\frac{1}{n}, \beta}^{\gamma, q . n N}(z)\right| \leq T
$$

where

$$
T=\left[\frac{(\gamma)_{q(N+1)}\left|z^{n}\right|^{N+1}}{\Gamma(\beta+N+1)(N+1) !}+\frac{(\gamma)_{q N}}{\Gamma(\beta+N)} \sum_{k=1}^{n-1} \frac{|z|^{k+n N}}{\Gamma\left(\frac{k+n N}{n}+1\right)}\right] E_{1, \beta}^{\gamma, q}\left(\left|\operatorname{Re} z^{n}\right|\right)
$$

Proof. From (1.7) and (1.8), we have

$$
E_{\frac{1}{n}, \beta}^{\gamma, q}(z)-E_{\frac{1}{n}, \beta}^{\gamma, q, n N}(z)=\left(E_{\frac{1}{n}, \beta}^{\gamma, q}\left(z^{n}\right)-\sum_{m=0}^{N} \frac{(\gamma)_{q m} z^{n m}}{\Gamma(m+\beta) m !}\right)+\sum_{m=0}^{\infty} \sum_{j=1}^{n-1} \frac{(\gamma)_{q m} z^{n m+j}}{\Gamma(m+\beta) \Gamma\left(\frac{n m+j}{n}+1\right)} .
$$

Consider,

$$
\sum_{j=1}^{n-1} \frac{n}{\Gamma\left(1-\frac{j}{n}\right)} \int_{0}^{z}\left\{E_{1, \beta}^{\gamma, q}\left(z^{n}-u^{n}\right)-\sum_{m=0}^{N-1} \frac{(\gamma)_{q m}\left(z^{n}-u^{n}\right)^{m}}{\Gamma(m+\beta) m !}\right\} u^{n-1-j} \mathrm{~d} u=\sum_{m=N}^{\infty} \sum_{j=1}^{n-1} \frac{n}{\Gamma\left(1-\frac{j}{n}\right)_{0}^{z}} \int_{0}^{z} \frac{(\gamma)_{q m}\left(z^{n}-u^{n}\right)^{m}}{\Gamma(m+\beta) m !} u^{n-1-j} \mathrm{~d} u,
$$

using (1.10), the above equation reduces to

$$
=\sum_{m=N}^{\infty} \sum_{j=1}^{n-1} \frac{z^{n m+n-j}(\gamma)_{q m}}{\Gamma\left(1-\frac{j}{n}\right) \Gamma(m+\beta) m !} B\left(1-\frac{j}{n}, m+1\right),
$$

Now, by involving (1.12), we have

$$
=\sum_{m=0}^{\infty} \sum_{j=1}^{n-1} \frac{(\gamma)_{q m} z^{n m+j}}{\Gamma(m+\beta) \Gamma\left(\frac{n m+j}{n}+1\right)} .
$$

by which we write

$$
E_{\frac{1}{n}, \beta}^{\gamma, q}(z)-E_{\frac{1}{n}, \beta}^{\gamma, q, n N}(z)=\left(E_{\frac{1}{n}, \beta}^{\gamma, q}\left(z^{n}\right)-\sum_{m=0}^{N} \frac{(\gamma)_{q m} z^{n m}}{\Gamma(m+\beta) m !}\right)+\sum_{j=1}^{n-1} \frac{n}{\Gamma\left(1-\frac{j}{n}\right)} \int_{0}^{z}\left\{E_{1, \beta}^{\gamma, q}\left(z^{n}-u^{n}\right)-\sum_{m=0}^{N-1} \frac{(\gamma)_{q m}\left(z^{n}-u^{n}\right)^{m}}{\Gamma(m+\beta) m !}\right\} u^{n-1-j} \mathrm{~d} u .
$$


Therefore, further we have,

$$
\begin{aligned}
\left|E_{\frac{1}{n}, \beta}^{\gamma, q}(z)-E_{\frac{1}{n}, \beta}^{\gamma, q, n N}(z)\right| \leq \mid & \left|E_{\frac{1}{n}, \beta}^{\gamma, q}\left(z^{n}\right)-\sum_{m=0}^{N} \frac{(\gamma)_{q m} z^{n m}}{\Gamma(m+\beta) m !}\right| \\
& +\left|\sum_{k=1}^{n-1} \frac{n z^{n-k}}{\Gamma\left(1-\frac{k}{n}\right)} \int_{0}^{z}\left\{E_{1, \beta}^{\gamma, q}\left(z^{n}-z^{n} w^{n}\right)-\sum_{m=0}^{N-1} \frac{(\gamma)_{q m} z^{n m}\left(1-w^{n}\right)^{m}}{\Gamma(m+\beta) m !}\right\} w^{n-1-k} \mathrm{~d} w\right| .
\end{aligned}
$$

Using the inequality (1.17), we have

$$
\left|E_{\frac{1}{n}, \beta}^{\gamma, q}(z)-E_{\frac{1}{n}, \beta}^{\gamma, q, n N}(z)\right| \leq \frac{(\gamma)_{q(N+1)}\left|z^{n}\right|^{N+1}}{\Gamma(\beta+N+1)(N+1) !} E_{\frac{1}{n}, \beta}^{\gamma, q}\left(\left|\operatorname{Re} z^{n}\right|\right)+M,
$$

where

$$
M=\left|\sum_{k=1}^{n-1} \frac{n z^{n-k}}{\Gamma\left(1-\frac{k}{n}\right)} \int_{0}^{1}\left\{E_{1, \beta}^{\gamma, q}\left(z^{n}-z^{n} w^{n}\right)-\sum_{m=0}^{N-1} \frac{(\gamma)_{q m} z^{n m}\left(1-w^{n}\right)^{m}}{\Gamma(m+\beta) m !}\right\} w^{n-1-k} \mathrm{~d} w\right|
$$

We see that,

$$
\begin{aligned}
M & \leq \sum_{k=1}^{n-1} \frac{n|z|^{n-k}}{\Gamma\left(1-\frac{k}{n}\right)} \int_{0}^{1} \frac{(\gamma)_{q N}\left|z^{n}\left(1-w^{n}\right)\right|^{N}}{\Gamma(\beta+N) N !} E_{1, \beta}^{\gamma, q}\left(\left|\operatorname{Re} z^{n}\left(1-w^{n}\right)\right|\right) w^{n-1-k} \mathrm{~d} w, \\
& \leq \sum_{k=1}^{n-1} \frac{(\gamma)_{q N}|z|^{n-k+n N}}{\Gamma(\beta+N) N ! \Gamma\left(1-\frac{k}{n}\right)} E_{1, \beta}^{\gamma, q}\left(\left|\operatorname{Re} z^{n}\right|\right) B\left(1-\frac{k}{n}, N+1\right),
\end{aligned}
$$

which on using (1.12), gives

$$
M \leq \frac{(\gamma)_{q N}}{\Gamma(\beta+N)} \sum_{k=1}^{n-1} \frac{|z|^{k+n N} E_{1, \beta}^{\gamma, q}\left(\left|\operatorname{Re} z^{n}\right|\right)}{\Gamma\left(\frac{k+n N}{n}+1\right)} .
$$

Inequalities (2.9) and (2.10) lead to the proof of the Theorem.

Remark on Theorem 4. If $\beta=\gamma=q=1$ then (2.7) reduces to an interesting inequality

$$
\left|E_{\frac{1}{n}}(z)-E_{\frac{1}{n}}^{n N}(z)\right| \leq \sum_{k=1}^{n-1} \frac{|z|^{k+n N} \exp \left(\operatorname{Re} z^{n}\right)}{\Gamma\left(\frac{k+n N}{n}+1\right)} .
$$

This inequality contains Mittag-Leffler and Exponential functions.

Recently, Shukla and Prajapati [9] obtained several properties of the function $E_{\alpha, \beta}^{\gamma, q}(z)$. The Mittag-Leffler function $E_{\alpha}(z)$ plays an important role in study of the various properties fractional calculus (Shukla and Prajapati [13]).

THEOREM 5. If $\alpha, \beta, \gamma, v \in \mathbb{C} ; \operatorname{Re}(\alpha)>0$,
$\operatorname{Re}(\beta)>0, \operatorname{Re}(\gamma)>0, \operatorname{Re}(v)>0$, and $q \in(0,1) \cup \mathbb{N}$, then

$$
\frac{1}{\Gamma(v)} \int_{0}^{z} t^{\beta-1}(z-t)^{v-1} E_{\alpha, \beta}^{\gamma, q}\left(\lambda t^{\alpha}\right) \mathrm{d} t=z^{\beta+v-1} E_{\alpha, \beta+v}^{\gamma, q}\left(\lambda z^{\alpha}\right) .
$$

Proof. Substituting $t=z u$ in left-hand side of (2.12) and then using (1.10) and (1.12), we get the required result.

Special cases of Theorem 5: For $\operatorname{Re}(v)>0$, from (2.12), we obtain several particular cases as listed below:

$$
\begin{gathered}
\frac{1}{\Gamma(v)} \int_{0}^{z}(z-t)^{v-1} e^{t} \mathrm{~d} t=z^{v} E_{1, v+1}^{1,1}(z), \\
\frac{1}{\Gamma(v)} \int_{0}^{z}(z-t)^{v-1} \cosh (\sqrt{t}) \mathrm{d} t=z^{v} E_{2, v+1}^{1,1}\left(z^{2}\right), \\
\frac{1}{\Gamma(v)} \int_{0}^{z}(z-t)^{v-1} \frac{\sinh (\sqrt{t})}{\sqrt{t}} \mathrm{~d} t=z^{v} E_{2, v+2}^{1,1}\left(z^{2}\right), \\
\frac{1}{\Gamma(v)} \int_{0}^{z}(z-t)^{v-1} \exp \left(z^{2}\right) e r f_{c}(-z) \mathrm{d} t=z^{v} E_{\frac{1}{2}, v+1}^{1,1}(z)
\end{gathered}
$$


THEOREM 6. If $\alpha, \beta, \gamma \in \mathbb{C} ; \operatorname{Re}(\alpha)>0, \operatorname{Re}(\beta)>0$, $\operatorname{Re}(\gamma)>0$ and $q \in(0,1) \cup \mathbb{N}$, then

$$
\begin{aligned}
& \frac{1}{\Gamma(\alpha)} \int_{0}^{z} t^{\beta-1}(z-t)^{\alpha-1} E_{2 \alpha, \beta}^{\gamma, q}\left(t^{2 \alpha}\right) \mathrm{d} t \\
& =z^{\beta-1}\left[E_{\alpha, \beta}^{\gamma, q}\left(z^{\alpha}\right)-E_{2 \alpha, \beta}^{\gamma, q}\left(z^{2 \alpha}\right)\right] .
\end{aligned}
$$

Proof. Consider the integral,

$$
\int_{0}^{z} E_{2 \alpha, \beta}^{\gamma, q}\left(t^{2 \alpha}\right) t^{\beta-1}\left\{1+\frac{(z-t)^{\alpha}}{\Gamma(1+\alpha)}\right\} \mathrm{d} t
$$

The above equation reduces to,

$$
\begin{aligned}
= & z^{\beta} \sum_{n=0}^{\infty} \frac{(\gamma)_{q n} z^{2 \alpha n}}{\Gamma(2 \alpha n+\beta+1) n !} \\
& +\sum_{n=0}^{\infty} \frac{(\gamma)_{q n}}{\Gamma(2 \alpha n+\beta) n !} \int_{0}^{1}(z u)^{2 \alpha n+\beta-1} \frac{z^{\alpha}(1-u)^{\alpha}}{\Gamma(1+\alpha)} z \mathrm{~d} u .
\end{aligned}
$$

Applying (1.10), (1.12) and further simplification of the above equation becomes,

$=z^{\beta} \sum_{n=0}^{\infty} \frac{(\gamma)_{q n} z^{2 \alpha n}}{\Gamma(2 \alpha n+\beta+1) n !}+z^{\beta} \sum_{n=0}^{\infty} \frac{(\gamma)_{q n} z^{(2 n+1) \alpha}}{\Gamma((2 n+1) \alpha+\beta+1) n !}$.

i.e.

$$
=z^{\beta} \sum_{n=0}^{\infty} \frac{(\gamma)_{q n} z^{\alpha n}}{\Gamma(\alpha n+\beta+1) n !} .
$$

Therefore,

$$
\int_{0}^{z} E_{2 \alpha, \beta}^{\gamma, q}\left(t^{2 \alpha}\right) t^{\beta-1}\left\{1+\frac{(z-t)^{\alpha}}{\Gamma(1+\alpha)}\right\} \mathrm{d} t=z^{\beta} E_{\alpha, \beta+1}^{\gamma, q}\left(z^{\alpha}\right) .
$$

Using Theorem 5 for $v=\lambda=1$, where the Equation (2.8) leads to

$$
\int_{0}^{z} E_{2 \alpha, \beta}^{\gamma, q}\left(t^{2 \alpha}\right) t^{\beta-1}\left\{1+\frac{(z-t)^{\alpha}}{\Gamma(1+\alpha)}\right\} \mathrm{d} t=\int_{0}^{z} t^{\beta-1} E_{\alpha, \beta}^{\gamma, q}\left(t^{\alpha}\right) \mathrm{d} t,
$$

differentiating above equation with respect to $z$, we get

$$
\begin{aligned}
& z^{\beta-1} E_{2 \alpha, \beta}^{\gamma, q}\left(z^{2 \alpha}\right)+\frac{\alpha}{\Gamma(1+\alpha)} \int_{0}^{z} t^{\beta-1}(z-t)^{\alpha-1} E_{2 \alpha, \beta}^{\gamma, q}\left(t^{2 \alpha}\right) \mathrm{d} t \\
& =z^{\beta-1} E_{\alpha, \beta}^{\gamma, q}\left(z^{\alpha}\right) .
\end{aligned}
$$

Thereby, the theorem is completely proved.

The theorem, that follows now, represents the relationship between the function $E_{\alpha, \beta}^{\gamma, q}(z)$ and the exponential function. This relationship presumably play an important role in the study of the fractional diffusion equation, i.e., heat conduction and mass transfer equation.
THEOREM 7. If $\alpha, \beta, \gamma \in \mathbb{C} ; \operatorname{Re}(\alpha)>0, \operatorname{Re}(\beta)>0$, $\operatorname{Re}(\gamma)>0$ and $q \in(0,1) \cup \mathbb{N}$, then

$$
\int_{0}^{\infty} e^{-\frac{x^{2}}{4 t}} E_{\alpha, \beta}^{\gamma, q}\left(x^{\alpha}\right) x^{\beta-1} \mathrm{~d} x=\sqrt{\pi} t^{\frac{\beta}{2}} E_{\frac{\alpha}{2}, \frac{\beta+1}{2}}^{\gamma, q}\left(t^{\frac{\alpha}{2}}\right) .
$$

Proof. Putting $z=\frac{\alpha n+\beta}{2}$ in (1.15), we have

$$
\frac{\Gamma\left(\frac{\alpha n+\beta}{2}\right)}{\Gamma(\alpha n+\beta)}=\frac{2 \sqrt{\pi}}{2^{\alpha n+\beta} \Gamma\left(\frac{\alpha n+\beta+1}{2}\right)} .
$$

The left-hand side of (2.19) gives,

$$
\begin{aligned}
& \int_{0}^{\infty} e^{-\frac{x^{2}}{4 t}} E_{\alpha, \beta}^{\gamma, q}\left(x^{\alpha}\right) x^{\beta-1} \mathrm{~d} x \\
& =\sum_{n=0}^{\infty} \frac{(\gamma)_{q n}}{\Gamma(\alpha n+\beta) n !} \int_{0}^{\infty} \mathrm{e}^{-\frac{x^{2}}{4 t}} x^{\alpha+\beta-1} \mathrm{~d} x,
\end{aligned}
$$

which leads to

$$
=\sum_{n=0}^{\infty} \frac{(\gamma)_{q n}}{\Gamma(\alpha n+\beta) n !} \frac{(2 \sqrt{t})^{\alpha n+\beta}}{2} \Gamma\left(\frac{\alpha n+\beta}{2}\right) .
$$

Use of (2.20) and further simplification of above equation yields

$$
=\sqrt{\pi} t^{\frac{\beta}{2}} \sum_{n=0}^{\infty} \frac{(\gamma)_{q n}}{\Gamma\left(\frac{\alpha}{2} n+\frac{\beta+1}{2}\right)} \frac{t^{\frac{\beta n}{2}}}{n !} .
$$

This completes the proof.

THEOREM 8. If $\alpha, \beta, \gamma \in \mathbb{C} ; \operatorname{Re}(\alpha)>0, \operatorname{Re}(\beta)>0$, $\operatorname{Re}(\gamma)>0$ and $q \in(0,1) \cup \mathbb{N}$ then

$$
\begin{aligned}
& \frac{\mathrm{d}}{\mathrm{d} x}\left[x^{\gamma-1} E_{\alpha, \beta}^{\gamma, q}\left(a x^{\alpha}\right)\right] \\
& =x^{\gamma-2}\left[E_{\alpha, \beta-1}^{\gamma, q}\left(a x^{\alpha}\right)+(\gamma-\beta) E_{\alpha, \beta}^{\gamma, q}\left(a x^{\alpha}\right)\right] .
\end{aligned}
$$

Proof. The left-hand side of (2.21) reduces to,

$$
\frac{\mathrm{d}}{\mathrm{d} x}\left[x^{\gamma-1} E_{\alpha, \beta}^{\gamma, q}\left(a x^{\alpha}\right)\right]=\sum_{n=0}^{\infty} \frac{(\gamma)_{q n} a^{n}(\alpha n+\gamma-1) x^{\alpha n+\gamma-2}}{\Gamma(\alpha n+\beta) n !} .
$$

The right-hand side can be expressed as

$$
\begin{aligned}
x^{\gamma-2}\left[\sum_{n=0}^{\infty}\right. & \frac{(\gamma)_{q n}(\alpha n+\beta-1) a^{n} x^{\alpha n}}{\Gamma(\alpha n+\beta) n !} \\
& \left.+\sum_{n=0}^{\infty} \frac{(\gamma)_{q n}(\gamma-\beta) a^{n} x^{\alpha n}}{\Gamma(\alpha n+\beta) n !}\right] .
\end{aligned}
$$


Involving (1.4), the proof is completed.

In a slightly different notation, the binomial theorem is

$$
(1-z)^{-\gamma, q}=\sum_{n=0}^{\infty} \frac{(\gamma)_{q n} z^{n}}{n !} .
$$

If $q=1$, then (2.22) reduces to simple binomial expression (Rainville [8]) that is,

$$
(1-z)^{-\gamma, 1}=(1-z)^{-\gamma}=\sum_{n=0}^{\infty} \frac{(\gamma)_{n} z^{n}}{n !} .
$$

THEOREM 9. If $\alpha, \beta, \gamma \in \mathbb{C} ; \operatorname{Re}(\alpha)>0, \operatorname{Re}(\beta)>0$, $\operatorname{Re}(\gamma)>0$ and $q \in(0,1) \cup \mathbb{N}$, then

$$
L^{-1}\left\{s^{-\beta}\left(1-\frac{z}{s^{\alpha}}\right)^{-\gamma, q}\right\}=t^{\beta-1} E_{\alpha, \beta}^{\gamma, q}\left(z t^{\alpha}\right) .
$$

Proof. Applying (2.22) to the left-hand side (2.23), we get

$$
\begin{aligned}
L^{-1}\left\{s^{-\beta}\left(1-\frac{z}{s^{\alpha}}\right)^{-\gamma, q}\right\} & =L^{-1}\left\{s^{-\beta} \sum_{n=0}^{\infty} \frac{(\gamma)_{q n}}{n !}\left(\frac{z}{s^{\alpha}}\right)^{n}\right\} \\
& =\sum_{n=0}^{\infty} \frac{(\gamma)_{q n} z^{n}}{n !} L^{-1}\left(\frac{1}{s^{\alpha n+\beta}}\right) \\
& =t^{\beta-1} E_{\alpha, \beta}^{\gamma, q}\left(z t^{\alpha}\right) .
\end{aligned}
$$

The proof is completed.

\section{Acknowledgements}

The authors would like to thank the reviewers for their valuable suggestions to improve the quality of paper.

\section{REFERENCES}

[1] R. Gorenflo, A. A. Kilbas and S. V. Rogosin, "On the Generalised Mittag-Leffler Type Function," Integral Transforms and Special Functions, Vol. 7, No. 3-4, 1998, pp. 215224. doi:10.1080/10652469808819200

[2] A. Wiman, "Uber de Fundamental Satz in der Theorie der
Funktionen $E_{\alpha}(x)$," Acta Mathematica, Vol. 29, No. 1, 1905, pp. 191-201. doi:10.1007/BF02403202

[3] T. R. Prabhakar, "A Singular Integral Equation with a Generalized Mittag-Leffler Function in the Kernel," Yokohama Mathematical Journal, Vol. 19, 1971, pp. 7-15.

[4] R. Gorenflo and F. Mainardi, "On Mittag-Leffler Function in Fractional Evaluation Processes," Journal of Computational and Applied Mathematics, Vol. 118, No. 1-2, 2000, pp. 283-299. doi:10.1016/S0377-0427(00)00294-6

[5] A. A. Kilbas and M. Saigo, "On Mittag-Leffler Type Function, Fractional Calculus Operators and Solution of Integral Equations," Integral Transforms and Special Functions, Vol. 4, No. 4, 1996, pp. 355-370. doi:10.1080/10652469608819121

[6] A. A. Kilbas, M. Saigo and R. K. Saxena, "Generalised Mittag-Leffler Function and Generalised Fractional Calculus Operators," Integral Transforms and Special Functions, Vol. 15, No. 1, 2004, pp. 31-49. doi:10.1080/10652460310001600717

[7] K. S. Miller, "The Mittag-Leffler and Related Functions," Integral Transforms and Special Functions, Vol. 1, No. 1, 1993, pp. 41-49. doi:10.1080/10652469308819007

[8] M. Saigo and A. A. Kilbas, "On Mittag-Leffler Type Function and Applications," Integral Transforms and Special Functions, Vol. 7, No. 1-2, 1998, pp. 97-112. doi:10.1080/10652469808819189

[9] A. K. Shukla and J. C. Prajapati, "On a Generalization of Mittag-Leffler Function and Its Properties," Journal of Mathematical Analysis and Applications, Vol. 336, No. 2, 2007, pp. 797-811. doi:10.1016/j.jmaa.2007.03.018

[10] M. Ikehata and S. Siltanen, "Electrical Impedance Tomography and Mittag-Leffler Function," Inverse Problems, Vol. 20, No. 4, 2004, pp. 1325-1348. doi:10.1088/0266-5611/20/4/019

[11] E. D. Rainville, "Special Functions," The Macmillan Company, New York, 1960.

[12] I. N. Sneddon, "The Use of Integral Transforms," Tata McGraw-Hill Publication Co. Ltd., New Delhi, 1979.

[13] A. K. Shukla and J. C. Prajapati, "On Generalized Mittag-Leffler Type Function and Generated Integral Operator," Mathematical Sciences Research Journal, Vol. 12, No. 12, 2008, pp. 283-290. 